\title{
Detection and modelling of time-dependent QTL in animal populations
}

\author{
Mogens S. Lund ${ }^{1 *}$, Peter SoRENSEN ${ }^{1}$, Per MADSEN $^{1}$, \\ Florence JAFFRÉZIC ${ }^{2}$ \\ ${ }^{1}$ Faculty of Agricultural Sciences, Department of Genetics and Biotechnology, \\ University of Aarhus, Research Center Foulum, P.O. Box 508830 Tjele, Denmark \\ ${ }^{2}$ UR337 Station de génétique quantitative et appliquée, INRA, 78350 Jouy-en-Josas, France
}

(Received 2 January 2007; accepted 3 September 2007)

\begin{abstract}
A longitudinal approach is proposed to map QTL affecting function-valued traits and to estimate their effect over time. The method is based on fitting mixed random regression models. The QTL allelic effects are modelled with random coefficient parametric curves and using a gametic relationship matrix. A simulation study was conducted in order to assess the ability of the approach to fit different patterns of QTL over time. It was found that this longitudinal approach was able to adequately fit the simulated variance functions and considerably improved the power of detection of time-varying QTL effects compared to the traditional univariate model. This was confirmed by an analysis of protein yield data in dairy cattle, where the model was able to detect QTL with high effect either at the beginning or the end of the lactation, that were not detected with a simple 305 day model.
\end{abstract}

QTL detection / longitudinal data / random regression models

\section{INTRODUCTION}

Detection of quantitative trait loci (QTL) has been an active field of research in animal genetics over recent years. Many of the traits of interest in these studies are measured repeatedly over time. In this paper "time" is used as a point along the trajectory of a longitudinal trait. Examples are milk production, fat and protein yields or somatic cell count for dairy cattle, growth curves for pigs or beef cattle, and age-specific fitness components such as survival and reproductive output.

In QTL mapping studies, longitudinal traits have generally been modelled as one record even though it is a function of several measurements recorded over a time period. This model fits the average QTL effect over time, which might be appropriate if the effect of the QTL is constant over time. However, QTL

*Corresponding author: Mogens.Lund@agrsci.dk

Article published by EDP Sciences and available at http://www.gse-journal.org or http://dx.doi.org/10.1051/gse:2007043 
that are differentially expressed over time often show a low average effect, and are as a consequence difficult to identify. Therefore, the statistical power to detect time-dependent QTL can be increased by using longitudinal models on repeated records.

There are various examples where the QTL effects are expected to change over time. In dairy cattle, for instance, the lactation starts with a rapid increase to a maximum production peak early in lactation and then declines gradually to the end of lactation. This reflects dramatic changes in the physiological state of dairy cattle during the lactation, with fluctuating concentrations of hormones, enzymes, and other components that are influencing milk production. It is likely that these biological components influence the QTL expression, which will result in non constant QTL effects over time. In fact evidence from polygenic studies suggest that the additive genetic variance changes over lactation stages for production traits in dairy cattle $[2,7,18,24]$.

Modelling these time-dependent QTL effects are relevant from a biological perspective of understanding the QTL's expression pattern over time, as well as for genetic selection purposes. For instance QTL that only affect milk yield in late lactation might be more valuable than QTL affecting milk yield only in the early peak lactation. This is because alleles that increase early peak lactation are likely to increase the physiological stress due to higher production and thereby the susceptibility to metabolic and reproductive disorders.

A few other authors have presented methods for longitudinal QTL modelling. Ma et al. [13], as well as $\mathrm{Wu}$ and Hou [25], proposed a methodology based on a maximum likelihood approach that requires a quite simple genetic structure of the data: either backcross, F2 or full-sib families. Rodriguez-Zas et al. [22] proposed to use a non-linear function to model individual production curves in dairy cattle. The parameters of this function have biological interpretation in terms of peak of production and persistency, and a QTL analysis was performed on these parameters using single-marker and interval mapping models. Moreno et al. [19] proposed a model for QTL detection in survival traits.

A longitudinal approach using random regression models for time-varying QTL has first been presented by Lund et al. [11] for animal populations and Macgregor et al. [14] in humans. Both model multi-allelic QTL using the random QTL effect model $[3,4]$. This method has some advantages over those previously presented in the literature. First, the direct modelling of QTL effects as a function of time is more flexible than modelling a QTL effect on the parameters of a specific parametric curve. Consequently, it can be more generally applied to different traits and can better model the process of specific 
genes being turned on and off. Secondly, basing the approach on the mixed model methodology using the IBD matrix enables the analysis of a range of different genetic structures. In particular, it can handle more general pedigrees, use linkage and linkage disequilibrium in a fine mapping context [17].

A simulation study was performed by Macgregor et al. [15] to assess QTLdetection power of this approach in human nuclear families. In their study they simulated a single highly polymorphic marker that was completely linked to the QTL.

In this paper we chose to focus on a similar longitudinal mixed model approach for genome scan in animal populations. The objectives of this study were (i) to assess the ability of the approach to fit different patterns of QTL effects over time in a simulated data set, (ii) to verify the hypothesis that the effects of QTL for protein production in dairy cattle generally change over time, and (iii) to verify the hypothesis that the power to identify a QTL is higher for the proposed method than with a traditional univariate method. This was investigated in a simulation study and a real example of protein yield in dairy cattle.

\section{MATERIALS AND METHODS}

As in the traditional quantitative genetics model for analysing functionvalued traits, it is assumed that the observed phenotypic character is a random variable $Y(t)$ and can be decomposed as:

$$
Y(t)=\mu(t)+g(t)+p(t)+e(t)
$$

where $\mu(t)$ are the fixed effects, which include the mean curve in the population, $p(t)$ are the permanent environmental effects and $e(t)$ is the residual term. The residuals are assumed to be independent but their variances can change with time. The genetic effects $g(t)$ are assumed to be decomposed into a sum of the QTL allelic effects $q_{i}(t)$ and the remaining polygenic effects $u(t)$ :

$$
g(t)=\sum_{i=1}^{N_{q t l}} q_{i}(t)+u(t) .
$$

The random variables $q_{i}(t), u(t)$ and $p(t)$ are assumed to be stochastic Gaussian processes, with mean zero and covariance functions $K_{i}(t, s), G(t, s)$ and $E(t, s)$ between times $t$ and $s$, respectively. In the equation above $N_{q t l}$ represents the total number of QTL with additive effects to be detected. In the examples below, this number of QTL will be equal to one but the model can readily be applied to a larger number of additive QTL effects. 
Random regression models [1] are based on a direct parametric modelling of the individual curves. The most commonly used functions of time are orthogonal polynomials that have interesting numerical properties, but any other parametric functions of time can be used. For a quadratic polynomial, the allelic effects of the $i$ th QTL for individual $k$ will be modelled as:

$$
q_{i k}(t)=a_{i k}+b_{i k} t+c_{i k} t^{2}=\boldsymbol{\Phi} \boldsymbol{q}_{i k}
$$

where $\boldsymbol{q}_{i k}=\left(a_{i k}, b_{i k}, c_{i k}\right)^{\prime}$ are random variables following a multivariate normal distribution with mean zero and covariance matrix $\boldsymbol{K}_{\mathbf{0} i}$ of dimension $(3 \times 3)$, and $\boldsymbol{\Phi}=\left(1, t, t^{2}\right)$. The covariance function for the $i$ th QTL will be deduced from the estimated covariance parameters of $\boldsymbol{K}_{\mathbf{0} i}$ and the time vectors [5,9] as $\boldsymbol{K}_{\boldsymbol{i}}=\boldsymbol{\Phi} \boldsymbol{K}_{\mathbf{0} i} \boldsymbol{\Phi}^{\prime}$. Different parametric functions can be used to model each effect of the model (QTL, polygenic and environmental effects). Likelihood ratio tests can be used to test the significance of the polynomial coefficients for each of these effects to determine the most appropriate order.

In matrix notations, the random regression mixed model including QTL effects assuming a homogeneous residual variance can be written as:

$$
y=X \beta+\sum_{i=1}^{N_{q t l}} W q_{i}+Z_{1} u+Z_{2} p+e
$$

where $\boldsymbol{y}$ is a vector of length $n$ with observations taken at different time points, $\boldsymbol{\beta}$ is a vector of effects describing the fixed curve over time, $\boldsymbol{X}$ is a design matrix relating fixed effects to records, $\boldsymbol{W} \boldsymbol{q}_{\boldsymbol{i}}, \boldsymbol{Z}_{\mathbf{1}} \boldsymbol{u}$ and $\boldsymbol{Z}_{\mathbf{2}} \boldsymbol{p}$ are the random deviations from the fixed curve due to allelic effects of the $i$ th QTL, polygenic and permanent environmental effects. Vector $\boldsymbol{q}_{i}$ is of dimension $2 N_{g} p_{1}$, where $N_{g}$ represents the number of animals included in the gametic matrix and $p_{1}$ the number of random regression coefficients used to model the QTL effect. Vector $\boldsymbol{u}$ is, as in classical polygenic analyses, of dimension $N_{a} p_{2}$, where $N_{a}$ is the number of animals in the relationship matrix and $p_{2}$ is the number of random regression coefficients used to model this polygenic effect. The permanent environmental vector $\boldsymbol{p}$ is of dimension $N_{p} p_{3}$, where $N_{p}$ is the number of animals with records and $p_{3}$ is the number of random regression coefficients used to model this permanent environmental effect. Matrices $\boldsymbol{W}, \boldsymbol{Z}_{\mathbf{1}}$, and $\boldsymbol{Z}_{\mathbf{2}}$ are design matrices with covariates of the curve. The random vectors $\boldsymbol{q}_{\boldsymbol{i}}, \boldsymbol{u}, \boldsymbol{p}$ and $\boldsymbol{e}$ are assumed to be independent of each other and to follow multivariate normal distributions: $\boldsymbol{q}_{\boldsymbol{i}} \mid M, c_{i} \sim M V N\left(0, \boldsymbol{K}_{\mathbf{0} i} \otimes \boldsymbol{Q}_{i} \mid M, c_{i}\right), \boldsymbol{u} \sim M V N\left(0, \boldsymbol{G}_{\mathbf{0}} \otimes \boldsymbol{A}\right), \boldsymbol{p} \sim M V N\left(0, \boldsymbol{P}_{\mathbf{0}} \otimes \boldsymbol{I}\right)$ and $\boldsymbol{e} \sim \operatorname{MVN}\left(0, \boldsymbol{I} \sigma_{e}^{2}\right)$, where $\boldsymbol{K}_{\mathbf{0} i}, \boldsymbol{G}_{\mathbf{0}}$ and $\boldsymbol{P}_{\mathbf{0}}$ are variance-covariance matrices among random regression coefficients. Matrix $\boldsymbol{A}$ is the additive genetic relationship matrix and $Q_{i} \mid M, c_{i}$ is the gametic relationship matrix of the allelic 
effects at the $i$ th QTL conditional on marker data $(M)$ and the position $\left(c_{i}\right)$ on the chromosome. The gametic relationship matrix was calculated by the recursive algorithm proposed by Wang et al. [23].

Calculation of the IBD matrices and REML estimation of variance components were obtained with the software package DMU [16]. Maximising a sequence of restricted likelihoods over a grid of specific positions provides a likelihood profile of the QTL position. QTL detection was performed with a likelihood ratio test at the most likely position.

\section{SIMULATION STUDY}

The aim of the simulation study was to assess the ability of longitudinal models to fit different patterns of QTL effects over time and to compare their power of detection to traditional univariate methods.

\subsection{Model used to simulate the data}

The simulated pedigree was based on a small granddaughter design consisting of 20 unrelated grandsires each having 20 sons (referred to as sires). The linkage map consisted of 11 biallelic marker loci with $10 \mathrm{cM}$ between each locus. A biallelic QTL was positioned in the midpoint between the third and fourth marker. In all loci, allele frequencies were assumed to be 0.5 . Information contained in the simulated marker map was close to a microsatellite map. For each sire, daughter yield deviations (DYD) were calculated at 55 time points. DYD were based on 100 daughters and each had 11 test-day records with 30-day intervals. Among the 100 daughters, 20 had their first test-day on days $5,10,15,20$, and 25 .

A cubic Legendre polynomial was used to simulate the polygenic effect for each sire, as well as the Mendelian effect of each daughter. Several different parametric functions were considered for the allelic effect over time of the QTL, as described below. The fixed curve was assumed constant and the model used to simulate the data can be written as:

$$
\operatorname{DYD}_{s}(t)=\frac{1}{20}\left(\sum_{l=1}^{20}\left(\boldsymbol{f}(t) \boldsymbol{q}_{s l}+\boldsymbol{\Phi}(t) \boldsymbol{u}_{s}+\boldsymbol{\Phi}(t) \boldsymbol{m}_{l}+\boldsymbol{e}_{s l}(t)\right)\right)
$$

where $\mathrm{DYD}_{s}(t)$ is the daughter yield deviation for sire $s$ at day $t$. The term $\boldsymbol{q}_{s l}$ is the effect of the paternally inherited QTL allele of daughter $l$, and $\boldsymbol{f}(t)$ is the parametric function of time used to describe the allelic effect over time. 
The additive polygenetic effect $\boldsymbol{u}_{s}(t)=\boldsymbol{\Phi}(t) \boldsymbol{u}_{s}$ and the Mendelian effect of daughter $l$ at time $t\left(\boldsymbol{m}_{l}(t)=\boldsymbol{\Phi}(t) \boldsymbol{m}_{l}\right)$ were simulated according to a random regression model, where $\boldsymbol{\Phi}(t)=\left(\phi_{0}(t), \phi_{1}(t), \phi_{2}(t), \phi_{3}(t)\right)$ are the coefficients of a normalised third order (i.e. cubic) Legendre polynomial at time $t$, and $\boldsymbol{u}_{s}=\left(u_{0 s}, u_{1 s}, u_{2 s}, u_{3 s}\right)$ and $\boldsymbol{m}_{l}=\left(m_{0 l}, m_{1 l}, m_{2 l}, m_{3 l}\right)$ are the associated random coefficients assumed to follow multivariate normal distributions. The residual term $\boldsymbol{e}_{s l}(t)$ was assumed to be normally distributed with mean zero and a constant variance over time.

Parameter values for the polygenic and Mendelian covariance functions, as well as for the residual variance, were those estimated by Jakobsen et al. [7] on a real data set on protein yield in dairy cattle.

Three different scenarios were simulated which differed in the pattern of the QTL effect over time. In the first scenario, the QTL effect was constant over time (Fig. 1a) and was assumed to be about $20 \%$ of the total genetic variance. In the second scenario, an initially large effect declined gradually, and the effect was minimal in the second half of the time period (Fig. 1b). An incomplete Gamma function was used to simulate this pattern. The average QTL effect was smaller than in the first scenario. In the third scenario, the effect of the initially positive allele declined gradually to become negative in the second half of the time period, while the initially negative allele became positive (Fig. 1c). A piece-wise incomplete Gamma function was used for this third scenario. The average QTL effect over the time period was equal to zero although the individual QTL allelic effects were quite large at the beginning and at the end of the period.

Figure $2 \mathrm{a}$ shows the variance functions of the QTL allelic effects in the three scenarios. The polygenic and residual variances were the same for all scenarios, and are shown in Figure 2b.

\subsection{Analysis of the simulated data}

For each scenario 100 replicates were simulated as shown above. Replicates were analysed using a random regression model with a cubic Legendre polynomial for QTL, polygenic and residual effects. In each replicate two likelihood ratio tests were performed to test if the QTL was identified using the random regression model and the traditional $305 \mathrm{~d}$ model. Under both models, the marker haplotypes were assumed known for grandsires, when the gameticrelationship matrix was calculated. The restricted log-likelihoods were maximised using an Average Information REML procedure [8]. The maximisation 

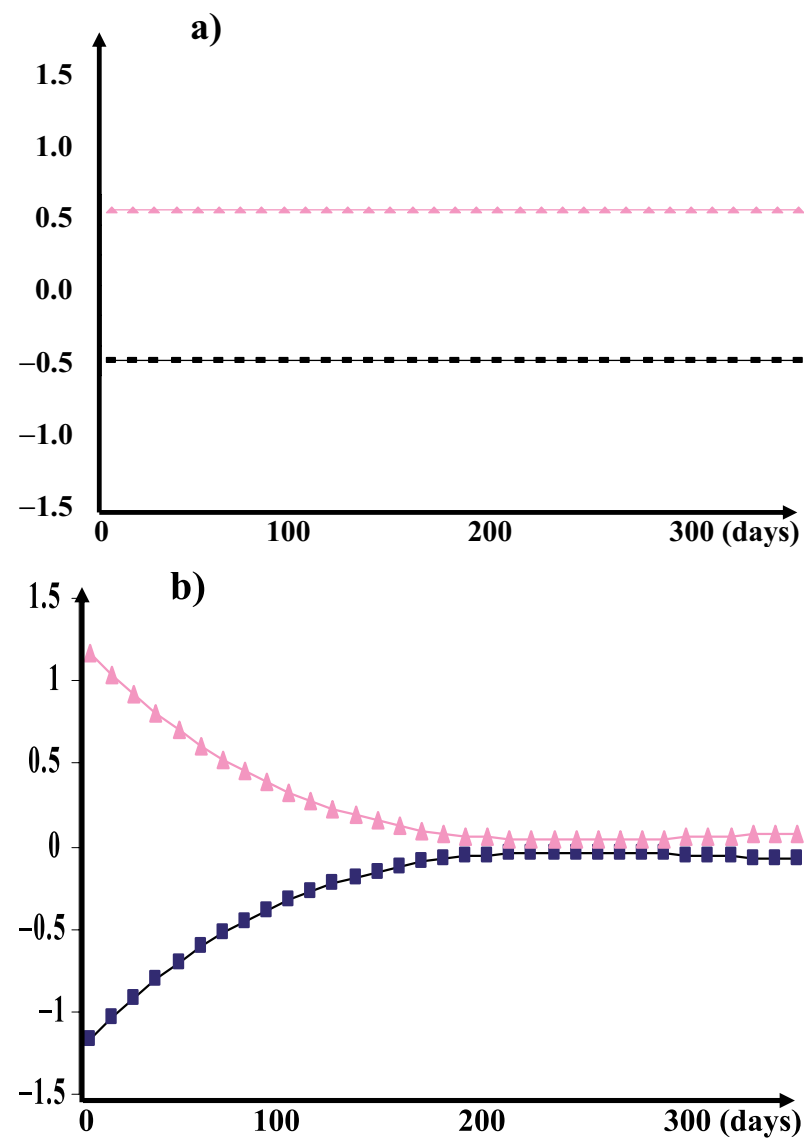

c)

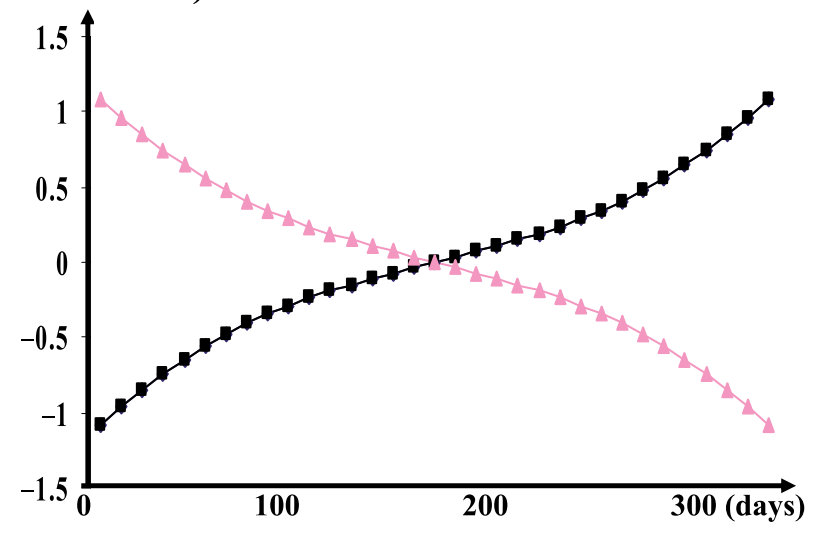

Figure 1. Effect of the two alleles of the biallelic QTL in the three scenarios for the simulated data. These effects are expressed as deviations from the fixed curve over time (days). 
a)

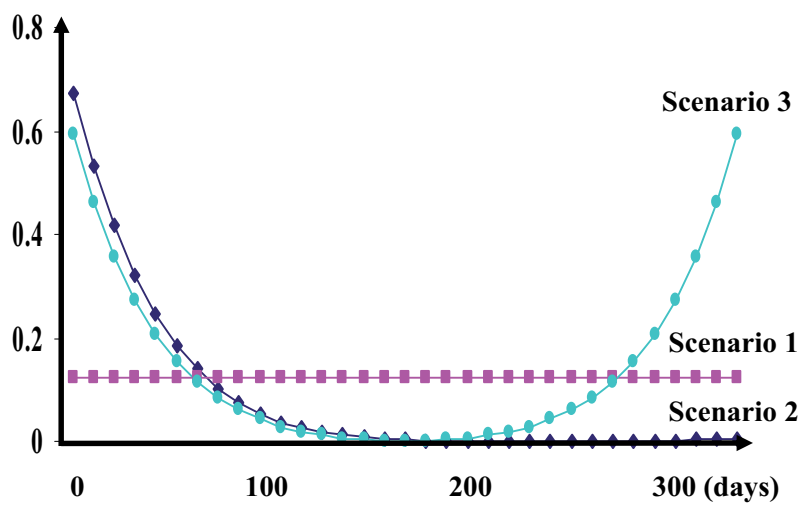

b)

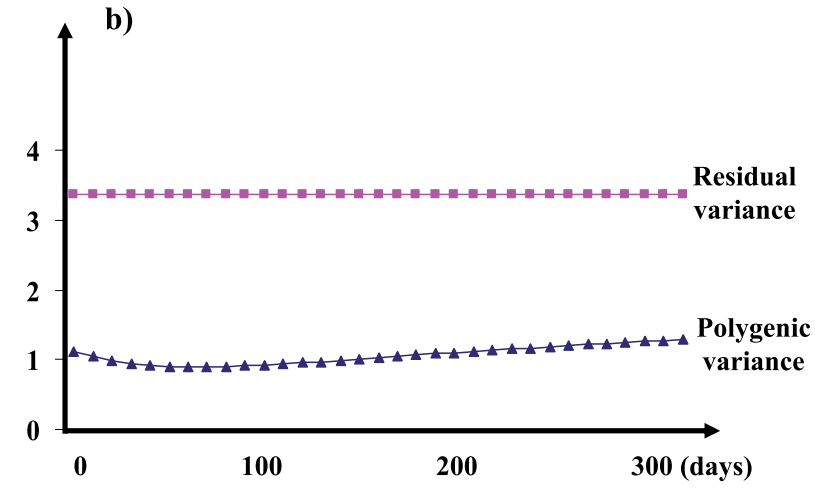

Figure 2. Variance function over time (days) in the three simulated scenarios due to the QTL (a) and residual and polygenic effects (b). These figures are on the same scale and can therefore be compared.

was performed every $3 \mathrm{cM}$ over the simulated $100 \mathrm{cM}$ interval. Data were analysed with a multiple allele model, although the simulated QTL was biallelic.

For the random regression model, the likelihood ratio test statistic was LRT1 $=\mathrm{L} 1 / \mathrm{L} 2$, where L1 and L2 are the maximum values of the restricted $\log$-likelihood under the models DYD $=\boldsymbol{\mu}+\boldsymbol{W} \boldsymbol{q}+\boldsymbol{Z} \boldsymbol{u}+\boldsymbol{e}$ and DYD $=\boldsymbol{\mu}+\boldsymbol{Z} \boldsymbol{u}+\boldsymbol{e}$, where $\boldsymbol{q}$ represents the QTL allelic effects, $\boldsymbol{u}$ the polygenic effect, $\boldsymbol{W}$ and $\boldsymbol{Z}$ are the incidence matrices. The polygenic effect was simulated according to a third order Legendre polynomial. It is therefore expected that this effect will be perfectly fitted with this random regression model. The QTL effect was simulated according to three different parametric functions of time, as presented above.

In the 305 dest, DYDmean for son $s$ was calculated as the mean of his 55 DYD. The likelihood ratio test statistic was LRT2 $=\mathrm{L} 3 / \mathrm{L} 4$, where L3 and 
Table I. Statistical power of tests for the QTL detection in the simulation study in the three different scenarios with a $305 \mathrm{~d}$ model and a third order random regression (i.e. number of times the simulated QTL was detected over 100 simulations).

\begin{tabular}{cccc}
\hline & Scenario 1 & Scenario 2 & Scenario 3 \\
\hline 305d & 62 & 16 & 6 \\
Random regression & 67 & 95 & 98 \\
\hline
\end{tabular}

L4 are the maximum values of the restricted log-likelihood under the models DYDmean $=\boldsymbol{\mu}+\boldsymbol{W} \boldsymbol{q}+\boldsymbol{Z} \boldsymbol{u}+\boldsymbol{e}$ and DYDmean $=\boldsymbol{\mu}+\boldsymbol{Z} \boldsymbol{u}+\boldsymbol{e}$. Under the 305d models the random polygenic and QTL allelic effects are multivariate normally distributed such as: $\boldsymbol{u} \sim \mathcal{N}\left(0, \sigma_{a}^{2} \boldsymbol{A}\right)$ and $\boldsymbol{q} \mid M, c \sim \mathcal{N}\left(0, \sigma_{q}^{2} \boldsymbol{Q} \mid M, c\right)$, where $M$ corresponds to the marker data and $c$ to the position on the chromosome. For each of the 100 simulated replicates, the test statistic was compared to the $5 \%$ empirical threshold found by simulation over 500 replicates under the null hypothesis.

\subsection{Results on simulated data}

The statistical power of the tests was calculated as the proportion of the 100 tests that were significant within each scenario and model type. As expected, when the QTL effect was constant over time the power of the two models was comparable. On the contrary, in scenarios 2 and 3, where the QTL effect was changing over time, the differences were substantial. In fact, as shown in Table I, for the second scenario where the QTL allelic effects were large during the first half of the period and nearly null during the second half, the QTL was detected in 95 percent of the cases with the random regression model and only 16 percent of the time with the $305 \mathrm{~d}$ model. The difference was even more pronounced for the third scenario where the average effect of the two alleles was zero, although each allele had an important effect varying during the whole time period. In this case, a considerable improvement was achieved using a longitudinal model compared to a 305d analysis. Indeed, the QTL was detected here in 98 percent of the cases with the random regression model and only 6 percent of the time with the $305 \mathrm{~d}$ model.

Given the estimated parameters of the random regression model, the variances can be calculated over time [9]. Figure 3 shows the average of the estimated curves of QTL variance over time over 100 replicates in the three scenarios, as well as the curves based on simulation input parameters. Differences observed for the first scenario are due to the fact that, in this case, since the QTL effect was assumed to be constant over time, a constant term 
a)

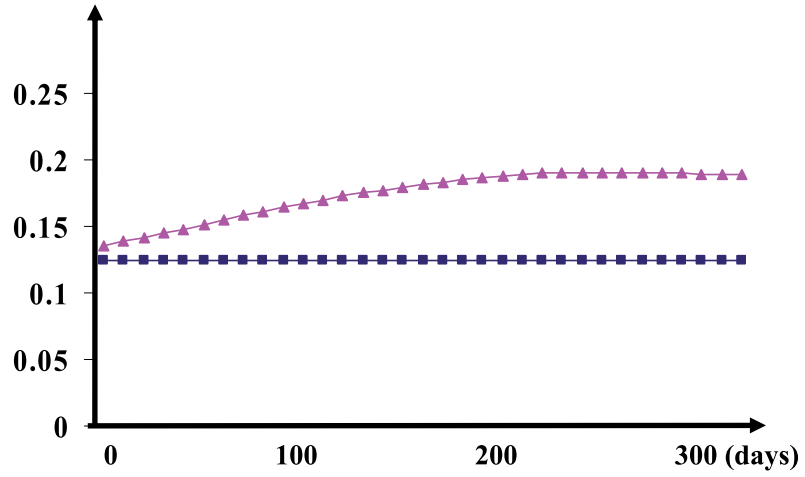

b)

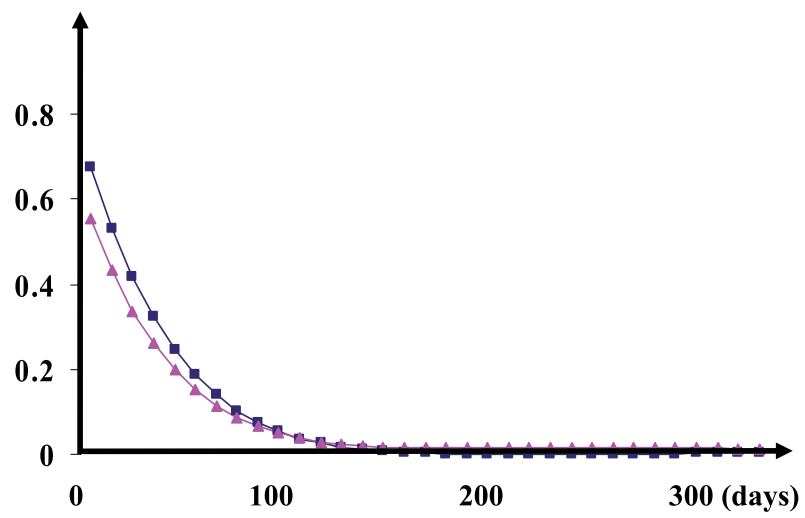

c)

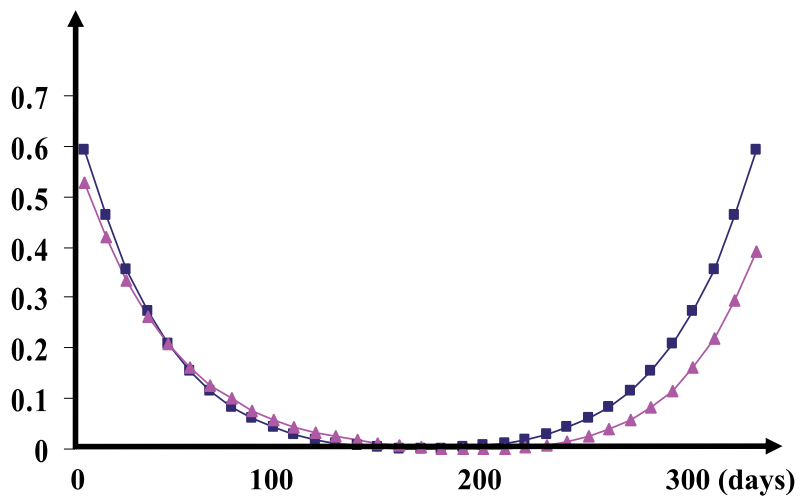

Figure 3. Curves of simulated (squares) and mean of estimated (triangles) variance functions of QTL allelic effects over time in scenario 1 (a), scenario 2 (b), and scenario 3 (c). 
would have been more appropriate than a cubic polynomial. In fact, as already shown in previous studies [5], high order polynomials tend to become extremely 'wiggly' and may not adequately fit simple covariance structures. In practical cases, a likelihood ratio test can be performed to determine the most appropriate order of the polynomial.

In the two other scenarios, however, when the QTL effect was assumed to change with time, the variances were well estimated with the random regression model. Indeed, for the second simulated scenario, Figure $3 \mathrm{~b}$ shows that the random regression model was able to predict that the QTL effect was the largest at the beginning of the time period. Similarly, Figure $3 \mathrm{c}$ shows that, for the third scenario, the model adequately predicts a larger QTL effect at the beginning and at the end of the time period. These estimations would be very useful for genetic selection.

It has to be pointed out, however, that the proposed longitudinal model will allow an increase of the detection power only if the QTL effect is sufficiently large at least during some parts of the time period. A time-varying QTL with a small effect during the whole lactation period will in fact not be detected. On the contrary, if the time-varying QTL has a quite large overall effect it will also be detected by the traditional $305 \mathrm{~d}$ model. In this case, the improvement reached with the longitudinal model will be the estimation of the QTL effect over time, which will allow to know during which parts of the time period it has the largest effect.

\section{APPLICATION ON REAL DATA}

In the dairy cattle breeding context, the use of test-day models to directly analyse monthly (or even daily) milk production measurements is now used in many different countries for genetic evaluation. It allows having more precise genetic value estimations but also to select for the shape of the lactation curve in order to improve, for instance, persistency. In these longitudinal models, the polygenic effect of each individual is assumed to be changing with time, as well as its variance and correlation functions. It is similarly expected in QTL analyses that these specific genes will also have an effect changing over time. Here, protein yield was used as an illustration of a longitudinal QTL detection analysis.

\subsection{QTL effect on protein yield in dairy cattle over the lactation}

A granddaughter design was used with 19 grandsires and 1394 sons. Seven chromosomes were scanned, which all had been previously reported in the 
literature to carry QTL for protein yield. For each chromosome, 4 to 12 markers were available. The exact names and positions of the markers for each chromosome are available upon request from the first author.

All production data from the Danish HF database were used to calculate DYD for each genotyped son. The model used for the DYD calculation is presented in detail by Lidauer et al. [10]. The only difference here is that the stage of lactation was modelled with fixed regression terms, as in Lidauer et al. [10], but nested within year*month of the test day. Two types of DYD for Danish Holstein bulls were produced. The first were based on 305 day records and produced one DYD per sire. The second were based on test day records and produced time dependent DYD in 10 day intervals, resulting in about 30 measurements along the lactation period (5 to $305 \mathrm{dim}$ ) for each sire.

Longitudinal QTL analyses were performed. Five different models were compared for QTL detection. All five models include fixed, polygenic and QTL effects. For the simple 305 day model, all the effects were assumed to be constant. Four different random regression models were then considered. For all of them, a third order Legendre polynomial was used for fixed and polygenic effects. Four different orders of Legendre polynomials were used for the QTL effect over time: from a simple random intercept to a cubic Legendre polynomial.

For each chromosome, the likelihood profile for the QTL detection was obtained by maximising the likelihood every $3 \mathrm{cM}$. At the most likely position on each chromosome a likelihood ratio test was performed. At present, a naive chi-square test was used since the mixture of chi-square correction is not readily applicable to compare the RR2 or RR3 model to the 'no QTL' model. The degrees of freedom of the tests were calculated as the difference between the number of parameters in the null model (with no QTL effect) and the longitudinal model. The results presented here will therefore be slightly too conservative, but this should not alter the main aim of this study which was to compare the 305 day model to various random regression models for QTL detection. The QTL variance functions were estimated over the lactation to see at which periods of time the QTL effect is likely to be the most important.

\subsection{Results}

Likelihood ratio test statistics for the seven scanned chromosomes for different orders of Legendre polynomials for the QTL effect are given in Table II. The chromosomes were chosen based on QTL detection for protein yield found in the literature. On this data set, however, the simple $305 \mathrm{~d}$ model 
Table II. Likelihood ratio test statistics of a 305 day model and random regression models from a simple random intercept (RR0) to a third order Legendre polynomial (RR3) for QTL effects. (Significant at 5\% (*) and 1\% (**) nominal levels from a chi-square test. nc: not converged.)

\begin{tabular}{cccccc}
\hline BTA & $305 d$ & RR0 & RR1 & RR2 & RR3 \\
\hline 9 & 0.9 & 0.0 & 0.6 & 9.3 & 12.4 \\
10 & 1.1 & 0.1 & $\mathbf{8 . 6}^{*}$ & 8.8 & 10.4 \\
14 & $\mathbf{3 . 9}^{*}$ & $\mathbf{7 . 9}^{* *}$ & $\mathbf{8 . 4}^{*}$ & nc & 7.2 \\
20 & 0.2 & 1.1 & $\mathbf{9 . 8}^{*}$ & 10.0 & 10.2 \\
23 & 0.1 & 0.0 & nc & nc & 5.2 \\
26 & 2.5 & 3.4 & 5.4 & 11.9 & 13.6 \\
27 & 0.0 & 1.1 & $\mathbf{9 . 8}^{*}$ & nc & 10.6 \\
\hline
\end{tabular}

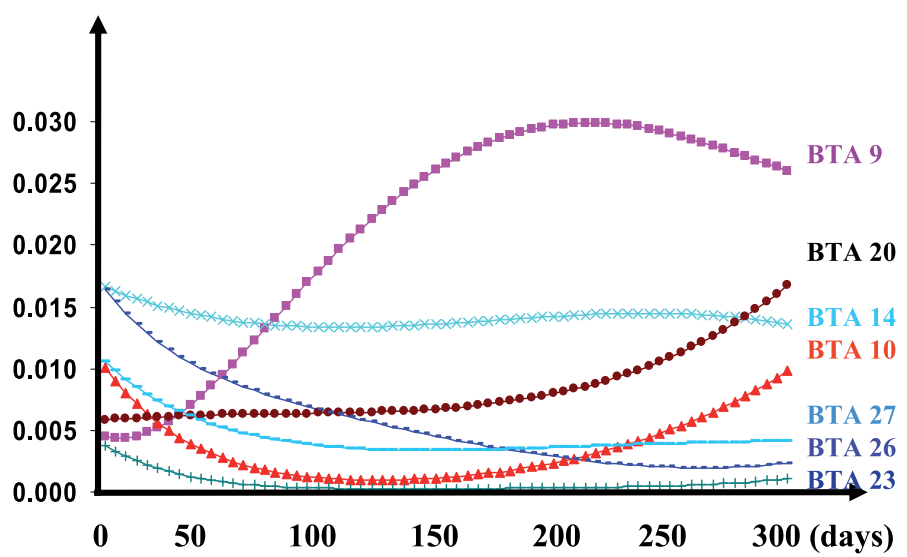

Figure 4. QTL variance functions over time at the maximum likelihood estimates for the seven chromosomes for the protein yield data in dairy cattle. QTL explained maximum $8 \%$ of the total genetic variance.

did not show enough power to detect any QTL for most of these chromosomes. It was found, in this example, that the use of longitudinal models allowed to increase the power of detection. This was especially observed on chromosomes 10, 20 and 27 for which QTL were not found significant with a 305 day model, but were detected with a first order Legendre polynomial. The QTL variance functions given in Figure 4 for these chromosomes show that the QTL on chromosome 20 has a higher effect at the end of the lactation than at the beginning and it is the contrary for the QTL detected on chromosome 27. For chromosome 10, the QTL has a quite large effect both at the beginning and the end of the period while it is very small in the middle of the lactation. 
As shown in Figure 4, many QTL were found to have a large effect either at the beginning or the end of the lactation period and very little over 305 days. For the first group, they could be genes that contribute to high physiological stress in the beginning of the lactation and might be most valuable as information on QTL with pleiotropic effects on disease resistance. Such pleiotropy will have to be verified in multiple trait analyses with functional traits. On the contrary, QTL with high effect at the end of the lactation will be more important for persistency.

On chromosome 14, the QTL that was already detected with a 305 day model was confirmed with the random regression model. Estimation of its variance over time (see Fig. 4), shows that its effect is large and nearly constant all over the lactation period. It has to be emphasised, however, that the DGAT1 region was not covered here with markers which explains why the likelihood ratio test statistics were not larger for this chromosome.

Table II shows that, for some QTL, the longitudinal effect was however quite difficult to model and required a high order polynomial, and therefore a large number of covariance parameters. This was especially the case for the QTL on chromosome 9 that was best described by a third order Legendre polynomial. Due to the large number of parameters involved, the likelihood ratio test for the presence of a QTL was not found significant although the effect of this QTL seems to be very large as shown in Figure 4. For this QTL, it might be useful to try using more parsimonious longitudinal models, although the shape of its variance and correlation functions might still be difficult to model with few parameters.

This study showed that the use of random regression models increased the power of detection of QTL that had a high effect for some parts of the lactation but not the whole period. They also allow to see in which time periods the effect of the QTL is the most important, which will be most useful for selection to improve the shape of the lactation curve. The large number of parameters involved in high order polynomials may, however, reduce the detection power when the QTL effect is not large enough. This can be observed for example in Table II for chromosome 14 with a third order Legendre polynomial. It is therefore important to choose the most adequate polynomial order for each QTL to avoid overfitting.

\section{DISCUSSION}

In QTL mapping studies, traits have often been defined as one record even though it is a function of several measurements recorded over a time period. 
An example is the 305 day milk yield, which is a weighted sum of a number of measurements recorded over the lactation. Using the sum may be reasonable if the genetic influence is constant over time. However, studies have shown that correlations between measurements differ over the lactation [2,24].

The results presented in this paper showed that the novel QTL-mapping method, based on longitudinal models, provides an appropriate and powerful tool to detect QTL affecting traits that are measured repeatedly over time. As shown in the simulation study, the power to detect QTL increases substantially compared to a standard 305d analysis, especially when the QTL-allelic effects change over time. This is because, in the $305 \mathrm{~d}$ model, the effect is averaged over the whole period, and may thereby not be large enough to be detected. On the contrary, the longitudinal models estimate the QTL variances and correlations at all time points and uses the information of the QTL effect in each part of the time period.

Analysis of the protein yield data in dairy cattle confirmed these results since QTL with high effect at the beginning or the end of the lactation and very low in the middle of the period were not detected with the simple 305 day model but were found significant with the longitudinal approach. The use of these models will therefore allow to detect new QTL that can have very important impact on persistency, for instance, which is of particular interest to breeders. Moreover, the methodology proposed here could be applied in marker assisted selection and would bring much more precision in the genetic values as more information is taken into account.

An issue still needs to be investigated, however, concerning the distribution of the likelihood ratio test statistics under the null hypothesis to detect the presence of the QTL. In fact, in the real data analysis presented here a naive chi-square distribution was assumed since the mixture of chi-square distributions are not known in the general case when a longitudinal QTL model is used. Permutations are usually recommended to determine the significance threshold but they can be in practice very computationally demanding and time-consuming. More theoretical research is therefore required to find the analytic distribution of the likelihood ratio test statistics for these longitudinal QTL models.

As shown in the real data analysis, high order polynomials may be required for the modelling of some QTL effects. In this case, the large number of parameters involved can prevent from detecting the QTL since the likelihood ratio test may not appear significant. This problem might be overcome by the use of more parsimonious longitudinal models such as character process $[5,20]$, 
or structured antedependence models [6]. This issue will be investigated in further studies.

Our approach can easily be extended to multiple QTL detection as investigated for single value characters by Lund et al. [12] as well as to the analysis of multiple correlated function-valued traits such as milk production, protein and fat contents. This QTL mapping approach can also be extended to the analysis of binary or categorical traits, as proposed by Pletcher and Jaffrézic [21] in polygenic models.

\section{CONCLUSION}

This study showed that the proposed model allowed to increase the power of QTL detection when the QTL effect was overall too small to be detected with classical methods but was still quite large during some part of the time period. The proposed methodology also allows to have a more precise estimation of the QTL effects over time. The longitudinal QTL approach therefore seems to be a promising area of research for future studies in livestock populations.

\section{ACKNOWLEDGEMENTS}

We thank the Danish Cattle Federation for providing phenotypic data and the Directorate for Food, Fisheries and Agri business for financial support.

\section{REFERENCES}

[1] Diggle P.J., Liang K.Y., Zeger S.L., Analysis of longitudinal data, Oxford University Press, 1994.

[2] Druet T., Jaffrézic F., Boichard D., Ducrocq V., Modeling lactation curves and estimation of genetic parameters for first lactation test-day records of French Holstein cows, J. Dairy Sci. 86 (2003) 2480-2490.

[3] Fernando R.L., Grossman M., Marker-assisted selection using best linear unbiased prediction, Genet. Sel. Evol. 21 (1989) 467-477.

[4] Grignola F.E., Hoeschele I., Tier B., Mapping quantitative trait loci in outcross populations via residual maximum likelihood, Genet. Sel. Evol. 28 (1996) 479-490.

[5] Jaffrézic F., Pletcher S.D., Statistical models for estimating the genetic basis of repeated measures and other function-valued traits, Genetics 156 (2000) 913-922.

[6] Jaffrézic F., Thompson R., Hill W.G., Structured antedependence models for genetic analysis of multivariate repeated measures in quantitative traits, Genet. Res. 82 (2003) 55-65. 
[7] Jakobsen J.H., Madsen P., Jensen J., Pedersen J., Christensen L.G., Sorensen D.A., Genetic parameters for milk production and persistency for Danish Holsteins estimated in random regression models using REML, J. Dairy Sci. 85 (2002) 1607-1616.

[8] Jensen J., Mantysaari E., Madsen P., Thompson R., Residual maximum likelihood estimation of (co)variance components in multivariate mixed linear models using average information, J. Indian Soc. Agric. Stat. 49 (1997) 215-236.

[9] Kirkpatrick M., Heckman N., A quantitative genetic model for growth, shape, reaction norms, and other infinite-dimensional characters, J. Math. Biol. 27 (1989) 429-450.

[10] Lidauer M., Pedersen J., Pösö J., Mäntysaari E.A., Strandén I., Madsen P., Nielen U.S., Eriksson J.-A., Johansson K., Aamand G.P., Joint Nordic Test Day Model: Evaluation Model, Interbull Bull. 35 (2006) 103-107.

[11] Lund M.S., Sorensen P., Madsen P., Linkage analysis in longitudinal data using random regression, Proc. 7th WCGALP, 32, 713-716, CD-rom Communication No 21-28, Montpellier, France, 2002.

[12] Lund M.S., Sorensen P., Guldbrandsten B., Sorensen D.A., Multitrait fine mapping of quantitative trait loci using combined linkage disequilibria and linkage analysis, Genetics 163 (2003) 405-410.

[13] Ma C.X., Casella G., Wu R., Functional mapping of quantitative trait loci underlying the character process: a theoretical framework, Genetics 161 (2002) 1751-1762.

[14] Macgregor S., Knott S.A., White I., Visscher P.M., Longitudinal variancecomponents analysis of the Framingham heart study data, BMC Genetics 4 (2003) S22, 5 p.

[15] Macgregor S., Knott S.A., White I., Visscher P.M., Quantitative trait locus analysis of longitudinal quantitative trait data in complex pedigrees, Genetics 171 (2005) 1365-1376.

[16] Madsen P., Sørensen P., Su G., Damgaard L.H., Thomsen H., Labouria R., DMU - a package for analyzing multivariate mixed models, Proc. 8th WCGALP, CD-rom Communication No 27-11, Belo Horizonte, Brazil, 2006.

[17] Meuwissen T.H.E., Goddard M.E., Fine mapping of quantitative traits using linkage disequilibria with closely linked marker loci, Genetics 155 (2000) 421-430.

[18] Meyer K., Grasser H.U., Hammond K., Estimates of genetic parameters for first lactation test day production of Australian black and white cows, Livest. Prod. Sci. 21 (1989) 177-199.

[19] Moreno C.R., Elsen J.M., Le Roy P., Ducrocq V., Interval mapping methods for detecting QTL affecting survival and time-to-event phenotypes, Genet. Res. 85 (2005) 139-149.

[20] Pletcher S.D., Geyer C.J., The genetic analysis of age-dependent traits: modeling a character process, Genetics 153 (1999) 825-833.

[21] Pletcher S.D., Jaffrézic F., Generalized character process models: estimating the genetic basis of traits that cannot be observed and that change with age or environmental conditions, Biometrics 58 (2002) 157-162. 
[22] Rodriguez-Zas S.L., Southey B.R., Heyen D.W., Lewin H.A., Detection of quantitative trait loci influencing dairy traits using a model for longitudinal data, J. Dairy Sci. 85 (2002) 2681-2691.

[23] Wang T., Fernando R.H., van der Beek S., van Arendonk J.A.M., Covariance between relatives for a marked quantitative trait locus, Genet. Sel. Evol. 27 (1995) 251-274.

[24] White I.M.S., Thompson R., Brotherstone S., Genetic and environmental smoothing of lactation curves with cubic splines, J. Dairy. Sci. 82 (1999) 632-638.

[25] Wu R., Hou W., A hyperspace model to decipher the genetic architecture of developmental processes: allometry meets ontogeny, Genetics 172 (2006) 627-637. 